\title{
Analysis of three-dimensional bone mineral density and bone strength measured by quantitative computed tomography following denosumab discontinuation in a patient with postmenopausal osteoporosis
}

This article was published in the following Dove Press journal: Clinical Interventions in Aging

\author{
Koki Tsuchiya' \\ Koji Ishikawa' \\ Soji Tani ${ }^{1}$ \\ Yusuke Oshita ${ }^{2}$ \\ Takuma Kuroda' \\ Ryo Yamamura' \\ Haruka Emori' \\ Hiroshi Maruyama' \\ Akira Matsuoka' \\ Yoshifumi Kudo' \\ Toshiyuki Shirahata' \\ Tomoaki Toyone' \\ Takashi Nagai' \\ Katsunori Inagaki \\ 'Department of Orthopaedic Surgery, \\ Showa University School of Medicine, \\ Tokyo 142-8666, Japan; ${ }^{2}$ Department of \\ Orthopaedic Surgery, Yamanashi Red \\ Cross Hospital, Yamanashi, Japan
}

\begin{abstract}
Discontinuation of denosumab during osteoporosis treatment leads to rapid loss of bone mineral density and induces a bone turnover rebound effect. Previous studies have reported analysis based on dual-energy X-ray absorptiometry scanning (DXA). Here, we report the first case involving analysis of three-dimensional bone mineral density and bone strength, measured by quantitative computed tomography (QCT) after discontinuation of denosumab. An 82-year-old woman who discontinued denosumab because of patient's wish was administered the fifth dose after a gap of 14 months. Her bone mineral density evaluated by DXA and QCT, bone strength, and bone turnover marker levels showed significant rebound phenomenon. The levels of the cortical parameters of the hip were also decreased indicating an increased risk of femoral fractures after denosumab interruption. Our case highlights the increased risk of fractures after discontinuation of denosumab. Therefore, denosumab must be used judiciously without interruption in the dosage schedule.
\end{abstract}

Keywords: lumbar spine, femoral neck, bone turnover markers, cortical bone, biomechanical analysis

\section{Introduction}

Denosumab is an entirely human monoclonal immunoglobulin G2 antibody that specifically binds to receptor activator of nuclear factor $\mathrm{\kappa B}$ ligand (RANKL), thereby, reversibly blocking bone resorption and leading to a decreased bone turnover, increased bone mineral density (BMD), and reduced fracture risk. ${ }^{1,2}$ Clinical studies indicate that the treatment of patients with osteoporosis with denosumab for a 10-year period leads to a continuous increase in the BMD, with a low incidence of adverse effects. ${ }^{3}$ Due to the high persistence with denosumab, promising results for osteoporosis treatment, and convenience of a 6-monthly injection regimen, ${ }^{4}$ denosumab is widely used. Despite its well-established efficacy for treating osteoporosis, several serious adverse effects of denosumab have been reported, ${ }^{5}$ including those due to treatment discontinuation. Discontinuation of denosumab leads to the rebound phenomenon, which involves an increase in bone turnover marker levels and decline in BMD. ${ }^{6,7}$ Several studies have reported rebound-associated multiple vertebral fractures using two-dimensional bone mineral density (2D-BMD) evaluation by dual-energy X-ray absorptiometry (DXA) ${ }^{7,8}$
Correspondence: Koji Ishikawa

Department of Orthopaedic Surgery,

Showa University School of Medicine, I-5-8

Hatanodai, Shinagawa, Tokyo I42-8666,

Japan

Tel +8I 337848543

Fax +8I 337849005

Email koji.ishikawa@med.showa-u.ac.jp 
However, to the best of our knowledge, no clinical study has been conducted to evaluate the effects of denosumab discontinuation using quantitative computed tomography (QCT). This is the first case report discussing the three-dimensional bone mineral density (3D-BMD) and bone strength after the discontinuation of denosumab.

\section{Case presentation}

The risk factors for osteoporosis in an 82-year-old woman were her age and a low body mass index (BMI). DXA performed in March 2015 revealed osteoporosis [lumbar spine (LS): $0.646 \mathrm{~g} / \mathrm{cm}^{2}$; femoral neck (FN): $\left.0.390 \mathrm{~g} / \mathrm{cm}^{2}\right]$ [T-score (LS): $-3.1 ;(\mathrm{FN}):-4.4][\mathrm{Z}$-score (LS): -0.6 ; (FN): -1.4]. The BMD was measured using a DXA scanner (Hologic QDR series: Hologic, Waltham, MA). All DXA measurements were analyzed by a radiologist at a central site. The 10-year probability of major osteoporotic fracture (MOF) evaluated by the fracture risk assessment tool (FRAX) was 34\%. The FRAX has been developed and validated by the World Health Organization to predict an individual 10-year risk of hip or major osteoporotic fractures. ${ }^{9}$ The patient was administered 60-mg denosumab injections every 6 months from March 2015 and responded well until the fourth dose. She was administered $100 \mathrm{mg}$ celecoxib and $100 \mathrm{mg}$ rebamipide as needed, but glucocorticoids was never received. She chose not to receive the fifth injection. The fifth dose was delayed and administered
14 months after the fourth injection. Compared to the parameters at the time of the fourth injection, her serum tartrateresistant acid phosphatase type 5 (TRAP-5b; the reference range in women: $120-420 \mathrm{mU} / \mathrm{dl}$; estimated by Osteolinks ${ }^{\circledR}$ TRAP-5b ${ }^{\circledR}$ Test Kit; DS Pharma Biomedical Co, Ltd, Osaka, Japan) and total serum procollagen I N-terminal propeptide (P1NP; the reference range in postmenopausal women: 26.4-98.2 $\mu \mathrm{g} / \mathrm{L}$; estimated by a total P1NP assay on the Elecsys automated analyzer; Roche Diagnostics, Basel, Switzerland) levels at the fifth injection showed significant rebound (TRAP-5b level: from $124 \mathrm{mU} / \mathrm{dl}$ to $1813 \mathrm{mU} / \mathrm{dl}$; total-P1NP level: from $13.6 \mu \mathrm{g} / \mathrm{L}$ to $175 \mu \mathrm{g} / \mathrm{L}$; Figure 1). Similarly, we noted a substantial decrease in the 2D-BMD, as measured by DXA (LS: from $0.759 \mathrm{~g} / \mathrm{cm}^{2}$ to $0.642 \mathrm{~g} / \mathrm{cm}^{2}$; FN: from $0.449 \mathrm{~g} / \mathrm{cm}^{2}$ to $0.327 \mathrm{~g} / \mathrm{cm}^{2}$; Figure 2) [T-score (LS): from -2.1 to -3.1 ; $(\mathrm{FN})$ : from -2.1 to -3.1$][\mathrm{Z}$-score (LS): from 0.1 to -0.6 ; (FN): from -0.7 to -1.9 ]. During the 3D-BMD analysis using QCT, a rapid decrease in BMD, including a decrease in the levels of the cortical parameters of the hip, was observed after denosumab discontinuation [LS: form $96.6 \mathrm{~g} / \mathrm{cm}^{3}$ to $88.1 \mathrm{~g} / \mathrm{cm}^{3}$; total hip (TH): from $594.1 \mathrm{~g} / \mathrm{cm}^{3}$ to $566.4 \mathrm{~g} / \mathrm{cm}^{3}$; cortical BMD: from $594.3 \mathrm{mg} / \mathrm{cm}^{3}$ to $566.5 \mathrm{mg} / \mathrm{cm}^{3}$; cortical thickness: from $1.146 \mathrm{~mm}$ to $0.956 \mathrm{~mm}$; Figures 3 and 4]. Furthermore, measurement of the biomechanical parameters of the hip revealed bone fragility after discontinuation of denosumab [a higher buckling ratio (BR), which is an index of cortical

\section{<Bone turnover markers>}

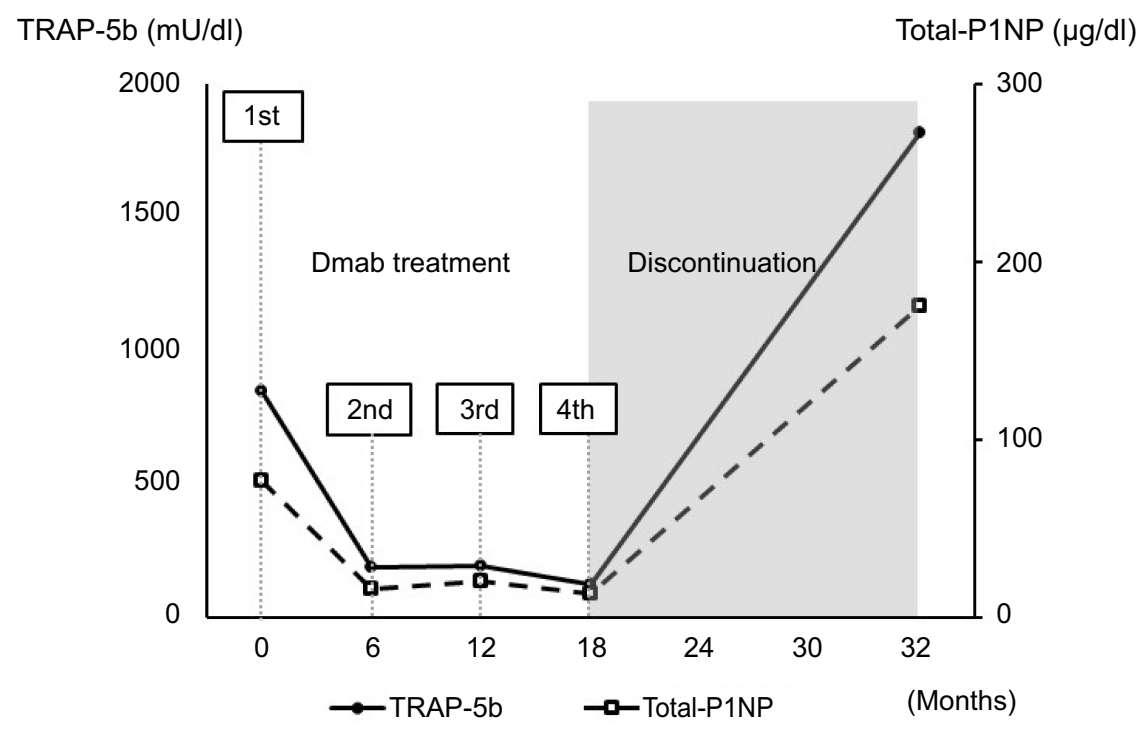

Figure I Bone turnover marker (TRAP-5b, total-PINP) levels. TRAP-5b and total-PINP levels increased immediately following discontinuation of denosumab. Abbreviations: Dmab, Denosumab; TRAP-5b, tartrate-resistant acid phosphatase type 5; total-PINP, total N-terminal propeptide of type I procollagen. 


\section{$<2$ D-Bone mineral density $>$}

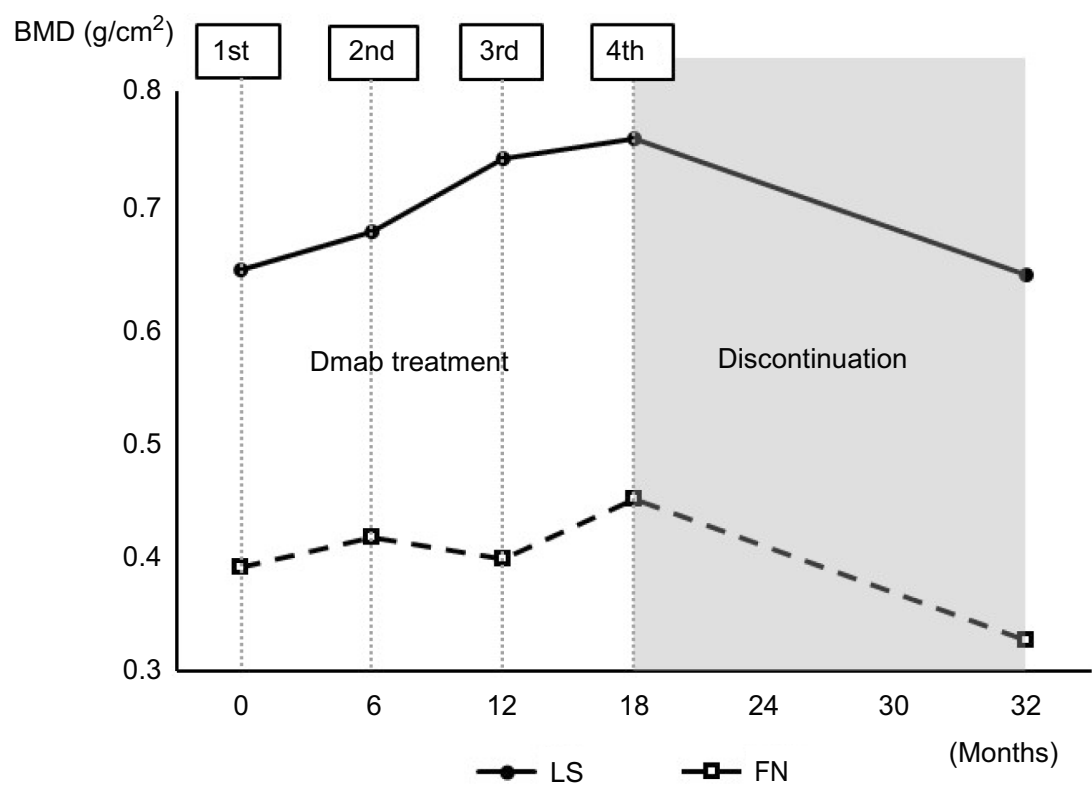

Figure 2 2D-BMD levels measured by DXA. DXA revealed that there was a significant decrease in bone mineral density after discontinuation of denosumab treatment. Abbreviations: 2D-BMD, two-dimensional bone mineral density; DXA, dual energy X-ray absorptiometry; Dmab, Denosumab; BMD, bone mineral density; LS, lumbar spine; FN, femoral neck.

instability (from 19.6 to 23.2 ) and a lower cross-sectional moment of inertia (CSMI), which is an index of bending (from $1.293 \mathrm{~cm}^{4}$ to $1.278 \mathrm{~cm}^{4}$ ); Figure 5]. The BR was calculated as the distance to the center of the bone mass divided by the average cortical thickness. ${ }^{10}$ The CSMI was defined by the integration of the products of incremental cross-sectional area and the square of their distance from the center of mass. ${ }^{11}$ Metabolic studies and tests aimed at ruling

\section{$<3$ D-Bone mineral density $>$}

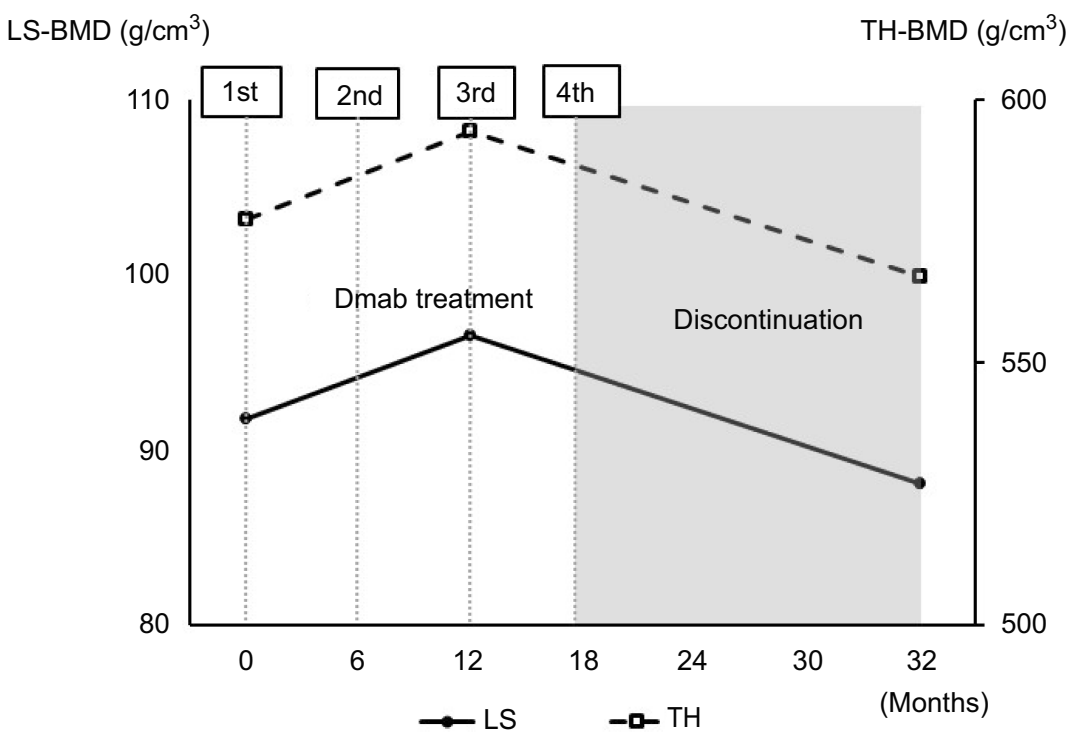

Figure 3 3D-BMD levels measured by QCT. QCT revealed that there was a significant decrease in bone mineral density after discontinuation of denosumab treatment. Abbreviations: 3D-BMD, three-dimensional bone mineral density; QCT, quantitative computed tomography; Dmab, denosumab; BMD, bone mineral density; LS, lumbar spine; $\mathrm{TH}$, total hip. 


\section{$<$ Cortical parameters of the hip $>$}

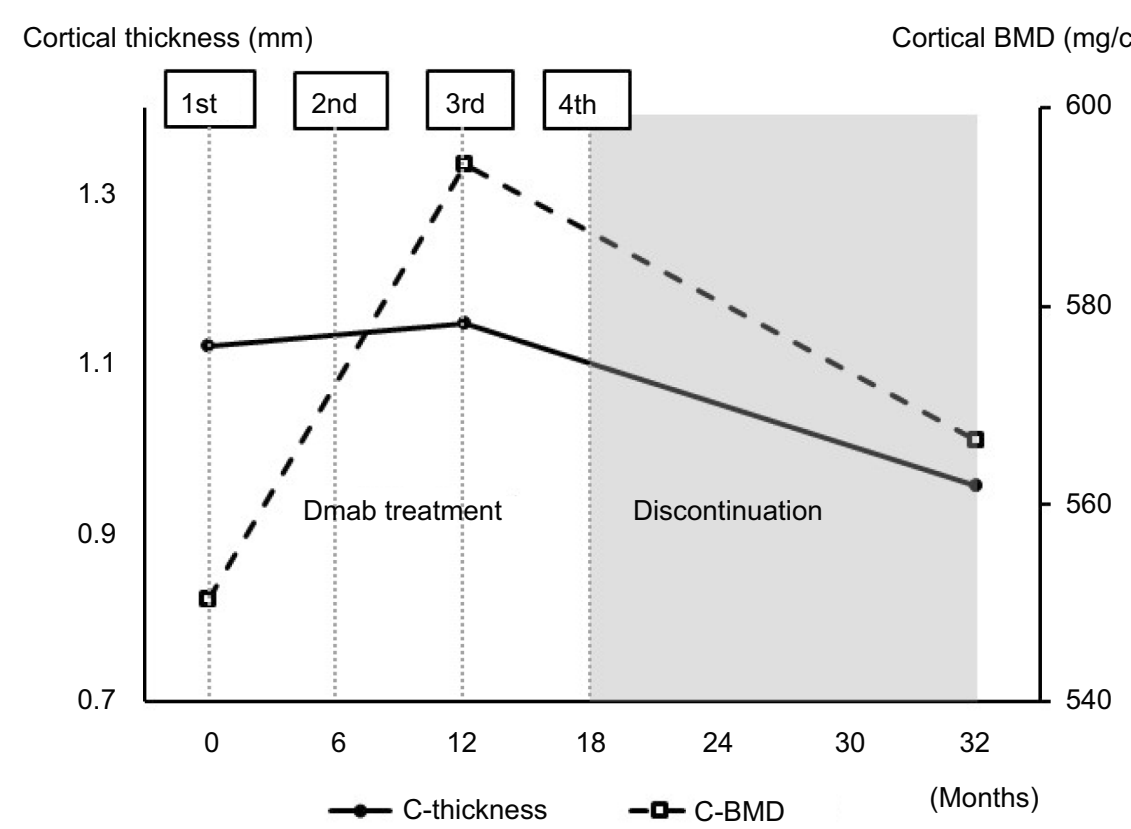

Figure 4 Cortical parameters of the hip measured by QCT. QCT revealed that the cortical parameter (cortical thickness and cortical BMD) decreased after discontinuation of denosumab treatment.

Abbreviations: QCT, quantitative computed tomography; Dmab, Denosumab; C-thickness, cortical thickness; C-BMD, cortical bone mineral density.

\section{<Biomechanical parameters of the hip $>$}

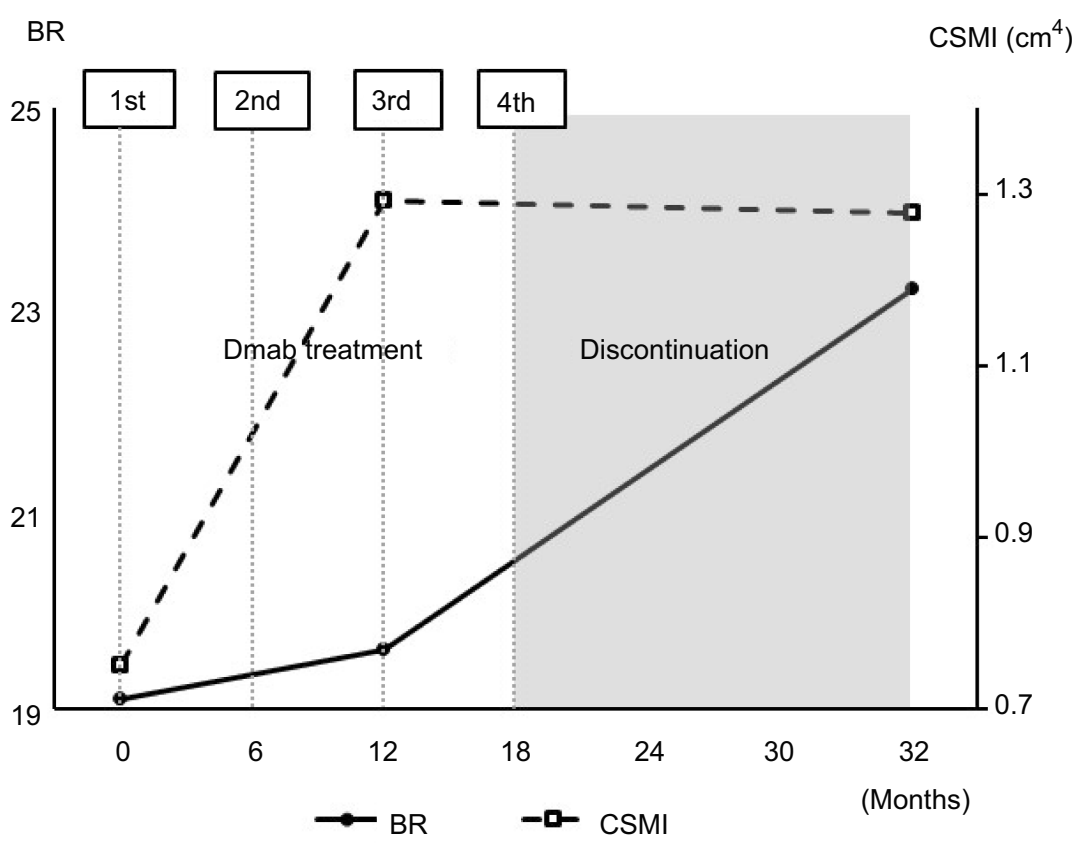

Figure 5 Biomechanical parameters of the hip measured by QCT. QCT revealed that the BR (index of cortical instability) increased and CSMI (index of bending strength) decreased after discontinuation of denosumab treatment.

Abbreviations: QCT, quantitative computed tomography; Dmab, Denosumab; BR, buckling ratio; CSMI, cross-sectional moment of inertia.

out potential secondary osteoporosis-related causes and other bone diseases, respectively, yielded normal results except for bone turnover markers (serum alkaline phosphatase level, $379.0 \mathrm{IU} / \mathrm{L}$; serum calcium level, $9.1 \mathrm{mg} / \mathrm{dl}$; 
serum phosphorus level, $3.4 \mathrm{mg} / \mathrm{dL}$; and serum estimated glomerular filtration rate, $51 \mathrm{~mL} / \mathrm{min}$ ). During treatment and after discontinuation of denosumab, she received adequate oral calcium (1000-1200 $\mathrm{mg} /$ day) and activated vitamin D (eldecalcitol: $0.75 \mu \mathrm{g} /$ day) supplementation.

\section{Consent}

The study was conducted with the approval of the ethics committee of the Yamanashi Red Cross Hospital and in accordance with the Declaration of Helsinki.

Written informed consent was obtained from the patient for the publication of this case report.

\section{Discussion}

In a long-term clinical study, denosumab treatment increased the BMD and decreased bone turnover for up to 10 years, with an acceptable safety profile. ${ }^{1}$ However, the efficacy of denosumab is reversible, and BMD increase at the LS and TH during 24 months of denosumab treatment were lost 1 year after the discontinuation of denosumab. ${ }^{7}$ Furthermore, recent case reports have raised concerns regarding the increased vertebral fragility after denosumab discontinuation, which suggests that patients should not stop taking denosumab, regardless of the BMD. ${ }^{7,8,12}$

Despite a number of well-established reports, ${ }^{12}$ it is unclear why only the incidence of multiple vertebral fractures increases. Notably, no study has reported femoral fractures after discontinuation of denosumab. The discrepancy between vertebral and non-vertebral fracture risk after denosumab discontinuation is perplexing. ${ }^{2}$ Considering that in the context of high bone turnover, BMD decreases rapidly following discontinuation of denosumab, it is expected that there is a strong association between trabecular bone and cortical bone. Eriksen et al has described that osteoclastic bone resorption is associated with shorter remodeling cycles during cortical bone resorption than during trabecular bone resorption. ${ }^{13}$ Therefore, the precise assessment of hip parameters, including the cortical and biomechanical parameters, is needed. In the present case, we demonstrated for the first time that there was a rapid decrease in the BMD measured by QCT in both the LS and hip, including a decrease in the levels of cortical parameters, after denosumab discontinuation. Furthermore, biomechanical parameters of the hip suggested fragility. DXA, which measures the 2D-BMD as a sum of all attenuating tissues in the beam projection, was used in all previous studies on denosumab discontinuation., ${ }^{7,8,12}$ Osteophytes may lead to overestimation of the BMD values assessed by DXA. ${ }^{14}$ Therefore, the previous reports may have underestimated the effect of denosumab discontinuation. With QCT, bone strength can be evaluated and trabecular bone and cortical bone can be assessed separately in three dimensions, without the interference of the surrounding osteophytes. ${ }^{9,15,16}$ The magnitude of bone loss was greater in the femoral bone than in the LS, and the FN-BMD returned to the average baseline value. These results are consistent with those of previous reports. ${ }^{17}$ Moreover, in the present case, it was observed that the levels of the cortical parameters of the hip also decreased. This may explain the fragility indicated by the biomechanical parameters (increased index of cortical instability and decreased index of bending strength) as the cortical bone is a major contributor to bone strength. While previous studies have reported that a higher BR and a lower CSMI are significantly associated with hip fractures, ${ }^{10}$ our patient fortunately did not develop a fracture. The discrepancy between a previous report that describes no decrease in the BMD at the FN assessed by DXA ${ }^{5}$ and our case could be due to the relatively long gap of 14 months in the administration of the fifth dose of denosumab with no vertebral fracture in our case. During the delayed interval in denosumab therapy, our patient presented with a transient acute increase in the TRAP-5b and total-P1NP levels. This finding is supported by the results of a previous study by Uebelhart et al, which reported that the highest bone turnover marker values were observed between 12 and 18 months after the administration of the last denosumab dose. ${ }^{18}$ The significant rebound effect of the present case (compared to the baseline, TRAP-5b was 2.14 times, and total-P1NP was 2.27 times value) might be related to the fact that the patient had never received any osteoporosis medication before starting denosumab. According to a previous study, patients previously treated with bisphosphonates have shown suppressed rebound effect after discontinuation. ${ }^{18}$ Even if denosumab treatment is discontinued, the use of another antiresorptive agent should be considered to prevent rapid bone loss and rebound phenomenon of bone turnover. ${ }^{19}$ However, no evidence-based treatment was available after the discontinuation of denosumab.

In conclusion, this is the first case reporting the 3D-BMD assessed by QCT and bone strength after the discontinuation of denosumab. Clinicians should not only consider cancellous bone fragility but also cortical bone fragility after the interruption of denosumab treatment. Our case highlights the risks associated with the discontinuation of denosumab. Denosumab must be suitably selected according to the patient's background and used judiciously without interruption in the dosage schedule. 


\section{Acknowledgment}

The authors thank Ayano Oyamada, Rikako Ono, Miho Mochizuki, Yoko Matsumoto, Kei Gonsho, Takeshi Eguro, and Keizo Sakamoto for their cooperation with osteoporosis treatment and technical assistance.

\section{Disclosure}

The authors report no conflicts of interest in this work.

\section{References}

1. Bone HG, Wagman RB, Brandi ML, et al. 10 years of denosumab treatment in postmenopausal women with osteoporosis: results from the phase 3 randomised FREEDOM trial and open-label extension. Lancet Diabetes Endocrinol. 2017;5(7):513-523. doi:10.1016/S2213-8587(17)30138-9

2. Anastasilakis AD, Polyzos SA, Makras P, Aubry-Rozier B, Kaouri S, Lamy O. Clinical features of 24 patients with rebound-associated vertebral fractures after denosumab discontinuation: systematic review and additional cases. J Bone Miner Res. 2017;32(6):1291-1296. doi: $10.1002 / \mathrm{jbmr} .3110$

3. Faienza MF, Chiarito M, D'amato G, et al. Monoclonal antibodies for treating osteoporosis. Expert Opin Biol Ther. 2018;18(2):149-157. doi:10.1080/14712598.2018.1401607

4. Karlsson L, Lundkvist J, Psachoulia E, Intorcia M, Ström O. Persistence with denosumab and persistence with oral bisphosphonates for the treatment of postmenopausal osteoporosis: a retrospective, observational study, and a meta-analysis. Osteoporos Int. 2015;26 (10):2401-2411. doi:10.1007/s00198-015-3253-4

5. Ishikawa K, Nagai T, Tsuchiya K, et al. High bone turnover status as a risk factor in symptomatic hypocalcemia following denosumab treatment in a male patient with osteoporosis. Clin Inter Aging. 2018;13:1929-1934. doi:10.2147/CIA.S180614

6. Popp AW, Zysset PK, Lippuner K. Rebound-associated vertebral fractures after discontinuation of denosumab-from clinic and biomechanics. Osteoporosis Int. 2016;27(5):1917-1921. doi:10.1007/s00198-015-3458-6

7. Niimi R, Kono T, Nishihara A, Hasegawa M, Kono T, Sudo A. Rebound-associated vertebral fractures after discontinuation of denosumab for the treatment of maxillitis. Osteoporosis Int. 2018;29 (3):769-772. doi:10.1007/s00198-017-4334-3

8. Aubry-Rozier B, Gonzalez-Rodriguez E, Stoll D, Lamy O. Severe spontaneous vertebral fractures after denosumab discontinuation: three case reports. Osteoporosis Int. 2016;27(5):1917-1921. doi:10.1007/s00198-015-3380-y
9. Kanis JA; on behalf of the World Health Organisation Scientific Group. Assessment of Osteoporosis at the Primary Health Care Level. WHO Collaborating Centre for Metabolic Bone Diseases, University of Sheffield; 2007. Available from: http://www.shef.ac. uk/FRAX/pdfs/WHO_Technical_Report.pdf. Accessed July 29. 2019.

10. Ito M, Wakao N, Hida T, et al. Analysis of hip geometry by clinical $\mathrm{CT}$ for the assessment of hip fracture risk in elderly Japanese women. Bone. 2010;46(2):453-457. doi:10.1016/j.bone.2009.08.059

11. Ito M, Nakamura T, Fukunaga M, Shiraki M, Matsumoto T. Effect of eldecalcitol, an active vitamin D analog, on hip structure and biomechanical properties: 3D assessment by clinical CT. Bone. 2011;49 (2):328-334. doi:10.1016/j.bone.2011.05.002

12. Bone HG, Bolognese MA, Yuen CK, et al. Effects of denosumab treatment and discontinuation on bone mineral density and bone turnover markers in postmenopausal women with low bone mass. $J$ Clin Endocrinol Metab. 2011;96(4):972-980. doi:10.1210/jc.2010-1502

13. Eriksen EF. Cellular mechanisms of bone remodeling. Rev Endocr Metab Discord. 2010;11(4):219-227. doi:10.1007/s11154-010-9153-1

14. Diederichs G, Engelken F, Marshall LM, et al. Diffuse idiopathic skeletal hyperostosis (DISH): relation to vertebral fractures and bone density. Osteoporos Int. 2011;22(6):1789-1797. doi:10.1007/s00198010-1409-9

15. Ishikawa K, Toyone $\mathrm{T}$, Shirahata $\mathrm{T}$, et al. A novel method for the prediction of the pedicle screw stability: regional bone mineral density around the screw. Clin Spine Surg. 2018;31(9):473-480. doi:10.1097/BSD.0000000000000703

16. Ishikawa K, Fukui T, Nagai T, et al. Type 1 diabetes patients have lower strength in femoral bone determined by quantitative computed tomography: a cross-sectional study. J Diabetes Investig. 2015;6 (6):726-733. doi:10.1111/jdi.12372

17. McClung MR, Wagman RB, Miller PD, Wang A, Lewiecki EM. Observations following discontinuation of long-term denosumab therapy. Osteoporosis Int. 2017;28(5):1723-1732. doi:10.1007/ s00198-017-3919-1

18. Uebelhart B, Rizzoli R, Ferrari SL. Retrospective evaluation of serum CTX levels after denosumab discontinuation in patients with or without prior exposure to bisphosphonates. Osteoporosis Int. 2017;28 (9):2701-2705. doi:10.1007/s00198-017-4080-6

19. Cummings SR, Ferrari S, Eastell R. Vertebral fractures after discontinuation of denosumab: a post hoc analysis of the randomized placebo-controlled FREEDOM trial and its extension. $J$ Bone Miner Res. 2018;33(2):188-189. doi:10.1002/jbmr.3337
Clinical Interventions in Aging

\section{Publish your work in this journal}

Clinical Interventions in Aging is an international, peer-reviewed journal focusing on evidence-based reports on the value or lack thereof of treatments intended to prevent or delay the onset of maladaptive correlates of aging in human beings. This journal is indexed on PubMed Central, MedLine, CAS, Scopus and the Elsevier
Bibliographic databases. The manuscript management system is completely online and includes a very quick and fair peer-review system, which is all easy to use. Visit http://www.dovepress.com/ testimonials.php to read real quotes from published authors. 